\title{
Efeitos de Manipulações de Propriedades Formais de Estímulos Verbais sobre o Comportamento
}

\author{
Mariella Vasconcelos Nogueira Braga \\ Luiz Carlos de Albuquerque ${ }^{1}$ \\ Carla Cristina Paiva Paracampo \\ Joene Vieira dos Santos \\ Universidade Federal do Pará
}

\begin{abstract}
RESUMO - Objetivando avaliar os efeitos de estímulos antecedentes verbais sobre o comportamento, 24 estudantes universitários foram expostos a um procedimento de escolha segundo o modelo; a tarefa consistia em apontar para cada um dos três estímulos de comparação. O comportamento correto era reforçado em razão fixa 6. Na Condição 1, a ordem de apresentação dos estímulos nas Fases 2, 3, 4 e 5 era: instrução correspondente, instrução mínima, pergunta correspondente, pergunta mínima, respectivamente. As oito condições diferiam quanto à ordem de apresentação das instruções e perguntas. A instrução e a pergunta correspondente estabeleceram o comportamento correto em $95 \%$ e $33 \%$ dos casos, respectivamente. A instrução e a pergunta mínima não estabeleceram o comportamento correto. Os resultados têm implicações para as definições de regras.
\end{abstract}

Palavras-chave: Definição de regras; efeitos de instruções; efeitos de perguntas.

\section{Effects of manipulation of formal properties of verbal stimuli on behavior}

\begin{abstract}
Aiming to investigate the effects of antecedent verbal stimuli on behavior, twenty-four students were exposed to a match to sample procedure. The task consisted of pointing out to each of three comparison stimuli. The correct behavior was reinforced in fixed ratio 6 . In Condition 1, the order of the presentation of the stimuli at the beginning of phases $2,3,4$ and 5 was: corresponding instruction, minimal instruction, corresponding question, minimal question, respectively. The eight conditions differed only in the order in which the verbal stimuli were presented. The corresponding stimuli and corresponding question established the correct behavior in $95 \%$ and $33 \%$ of the cases, respectively. The minimal stimuli didn't establish the correct behavior. The results have implications on the defining of rules.
\end{abstract}

Keywords: Defining rules; effects of instructions; effects of questions.

Ao fazer a distinção entre o comportamento governado por regras e o comportamento modelado por contingências, Skinner $(1963,1969,1974)$ definiu regras como estímulos discriminativos especificadores de contingências. Nem todos os autores, no entanto, têm concordado com essa proposição. Schlinger e Blakely (1987), por exemplo, argumentam que regras não funcionam como estímulos discriminativos, porque elas não evocariam comportamento. Regras, para estes autores, funcionariam como estímulos alteradores de função de outros estímulos. Já Zettle e Hayes (1982) argumentam que a proposição de Skinner não deixa claro o que significa especificar contingências e desconsidera exemplos de regras que não especificam claramente contingências de reforço.

Zettle e Hayes (1982) propuseram, então, que regras deveriam ser definidas como estímulos antecedentes verbais. Mas essa proposição de Zettle e Hayes não deixa claro se qualquer estímulo antecedente verbal poderia funcionar como

1 Endereço para correspondência: Universidade Federal do Pará. Rua Augusto Corrêa, 01. Núcleo de Teoria e Pesquisa do Comportamento. CEP: 66075-110. Guamá - Belém, PA. (91) 41414158. E-mail:1calbu@ufpa.br. regra, independentemente de sua forma. ${ }^{2}$ Isto considerando que um estímulo funcionaria como regra quando ele pudesse estabelecer comportamentos novos independentemente de suas consequências imediatas, que seria uma das suas principais funções (Albuquerque, 2001, 2005; Albuquerque, de Souza, Matos \& Paracampo, 2003). Por essa proposição de Albuquerque e colaboradores, dizer que regras estabelecem comportamento não implica que o comportamento estabelecido seja controlado por regras. O comportamento estabelecido por uma regra pode ser classificado de controlado por regras, quando ele ocorre independentemente de suas consequências imediatas (Albuquerque, 2001; Albuquerque, \& cols. 2003;

2 A expressão propriedades formais de estímulos verbais está sendo usada para se referir às características apresentadas pelo estímulo verbal que determinam, em parte, o que ele parece para uma comunidade verbal, de acordo com as suas práticas. Por exemplo, os estímulos: "você deve fazer?", "você deve fazer", "você deve fazer o dever" e "você deveria fazer o dever", apresentam algumas características que permitem que uma determinada comunidade possa dizer que o primeiro tem a forma de uma pergunta, o segundo tem a forma de uma ordem, o terceiro tem a forma de uma regra e essa regra tem a forma de uma ordem e o quarto tem a forma de uma regra e essa regra tem a forma de uma sugestão. 
Albuquerque, Matos, de Souza \& Paracampo, 2004; Albuquerque, Reis \& Paracampo, 2008).

Outro problema para a definição de regras como estímulos antecedentes verbais é que há evidências experimentais mostrando que os efeitos de estímulos antecedentes verbais dependem, em parte, de suas propriedades formais (Albuquerque \& Ferreira, 2001; Braga, Albuquerque \& Paracampo, 2005; Silva \& Albuquerque, 2006). Por exemplo, Silva e Albuquerque compararam o desempenho de participantes expostos a perguntas, que não especificavam o comportamento que produzia reforço, com o desempenho de participantes não expostos a perguntas. Observaram que perguntas que não especificam o comportamento que produz reforço podem facilitar o estabelecimento de um comportamento, mas não determinam a sua forma inicial. Já Braga e colaboradores compararam o desempenho de participantes expostos a instruções com o desempenho de participantes expostos a perguntas, quando as instruções e as perguntas especificavam o comportamento que produzia reforço. Observaram que, diferente de perguntas que não especificam o comportamento que produz reforço, perguntas que especificam esse comportamento, tal como instruções, podem exercer a função de estabelecer comportamentos novos.

Contudo, ainda não está claro que características um estímulo antecedente verbal deve apresentar para ele poder exercer a função de estabelecer comportamentos novos. Ainda não está claro, por exemplo, se o que é crítico para um estímulo antecedente verbal exercer essa função é o fato de ele especificar, ou não, o comportamento a ser estabelecido (o comportamento que produz reforço, no caso do presente estudo), ou é o fato de ele ser apresentado na forma de pergunta ou na forma de instrução.

A investigação de questões como estas também se justifica, porque, na área que investiga o controle por regras, poucos estudos (Albuquerque \& Ferreira, 2001; Braga \& cols., 2005; Degrandpre, Buskist \& Cush, 1990; England \& Buskist, 1995; Navarick, 2004; Silva \& Albuquerque, 2006) têm manipulado as propriedades formais dos estímulos verbais, isto é, poucos estudos têm manipulado os estímulos que constituem a regra. A maior parte dos estudos nessa área tem enfatizado a manipulação das consequências programadas para o comportamento de seguir regras (Barret, Deitz, Gaydos \& Quinn, 1987; Cerutti, 1991; Galizio, 1979; Hayes, Brownstein, Zettle, Rosenfarb \& Korn, 1986; Joyce \& Chase, 1990; Newman, Buffington \& Hemmes, 1995; Otto, Torgrud \& Holborn, 1999; Paracampo, Albuquerque, Farias, Carvalló \& Pinto, 2007; Shimoff, Catania \& Matthews, 1981; Weiner, 1970) ou das variáveis históricas que antecedem o comportamento de seguir regras (Albuquerque \& cols. 2003; DeGrandpre \& Buskist, 1991; LeFrancois, Chase \& Joyce, 1988; Martinez \& Tomayo, 2005; Pinto, Paracampo \& Albuquerque, 2006; Torgrud \& Holborn, 1990). No entanto, investigações que manipulem as propriedades formais de estímulos antecedentes verbais (como, por exemplo, investigações que comparem os efeitos de estímulos apresentados na forma de pergunta com os efeitos de estímulos apresentados na forma de instrução, e de estímulos que especificam e que não especificam o comportamento que produz reforço) também podem ser importantes, na medida em que contribuam para esclarecer qual o papel de tais propriedades formais na determinação dos efeitos dos estímulos antecedentes verbais (Albuquerque \& Ferreira, 2001).

Diante disso, o presente estudo procurou comparar os efeitos, sobre o comportamento não-verbal, de estímulos antecedentes verbais que especificam o comportamento não-verbal que produz reforço com os efeitos de estímulos antecedentes verbais que não especificam tal comportamento, quando: 1) estes estímulos são apresentados, sucessivamente, ora na forma de pergunta, ora na forma de instrução; 2) o comportamento não-verbal é reforçado em esquema de razão fixa 6 (FR6); e, 3) a sensibilidade deste comportamento a mudanças nas contingências de reforço programadas no experimento é testada. Neste esquema FR 6, cada seis emissões consecutivas de uma mesma sequência de respostas correta produzia um ponto. Erros ou a não emissão consecutiva de uma mesma sequência de respostas correta, reiniciavam a FR6 para a obtenção de ponto. Este esquema foi usado para garantir que o comportamento tivesse que persistir, antes de poder ser reforçado. Deste modo, poder-se-ia avaliar se uma instrução ou uma pergunta poderiam estabelecer um comportamento antes que as suas consequências programadas pudessem exercer qualquer efeito sobre ele.

No presente estudo, então, o desempenho de cada participante foi exposto a cinco fases. A primeira fase era de linha de base. Cada uma das demais quatro fases era constituída de duas sessões e cada sessão era encerrada após a ocorrência de 80 tentativas. Dentro de cada uma dessas quatro fases, as contingências programadas na primeira sessão sempre eram alteradas, sem sinalização, na segunda sessão. No início de cada uma dessas quatro fases era apresentado um estímulo antecedente verbal, que podia ser uma instrução que especificava o comportamento que produzia reforço, ou uma instrução que não especificava tal comportamento, ou uma pergunta que especificava o comportamento que produzia reforço, ou ainda uma pergunta que não especificava tal comportamento. A ordem em que estes estímulos foram apresentados foi manipulada entre condições experimentais apenas para avaliar efeitos de ordem.

Assim, se os estímulos antecedentes verbais podem exercer os seus efeitos independentemente de suas propriedades formais, como sugere a proposição de que regras são estímulos antecedentes verbais (Hayes, 1986; Zettle \& Hayes 1982), então, deveria ser esperado que qualquer um dos estímulos antecedentes verbais apresentados ao participante, independentemente de sua forma (isto é, independentemente de ele ser apresentado na forma de pergunta ou de instrução e de especificar, ou não, o comportamento que produz reforço) pudesse exercer a função de regras de estabelecer comportamentos novos independentemente de suas consequências imediatas (Albuquerque, 2005). Alternativamente, se os estímulos antecedentes verbais podem exercer os efeitos dependendo de suas propriedades formais, como sugere a proposição de que regras são estímulos especificadores de contingências (Skinner, 1969), então, deveria ser esperado que apenas os estímulos antecedentes verbais que especificassem o comportamento a ser estabelecido pudessem exercer essa função de regras. O presente estudo procurou avaliar essas possibilidades. 


\section{Método}

\section{Participantes}

Os participantes foram 24 estudantes universitários, sem história experimental prévia, matriculados em diferentes cursos (exceto o curso de Psicologia) e em diferentes semestres. Todos foram convidados a participar do experimento, atendendo a convite verbal do experimentador.

\section{Equipamentos e Material}

Foi utilizada uma mesa de madeira, medindo $150 \mathrm{x} 78 \mathrm{x}$ $70 \mathrm{~cm}$. Fixado à mesa, de modo a dividi-la ao meio em todo o seu comprimento, havia um anteparo com espelho unidirecional de $150 \times 60 \mathrm{~cm}$, fixado em uma moldura de madeira e localizado $13 \mathrm{~cm}$ acima do tampo da mesa. No centro do anteparo, junto ao tampo da mesa, havia uma abertura retangular de $45 \times 3 \mathrm{~cm}$. Acima e ao centro desta abertura, havia um contador operado pelo experimentador e com os dígitos voltados para o participante. Visível ao participante estava instalada no anteparo uma lâmpada transparente de 7 watts. Uma etiqueta de papel com a frase impressa: "Você ganhou um ponto" estava colada no anteparo, acima desta lâmpada. Uma lâmpada fluorescente de 15 watts estava instalada na borda superior e ao centro do anteparo. Ao lado do experimentador, havia uma fita cassete, um amplificador e um tape-deck. Dois fones de ouvido estavam conectados ao tape-deck. Um desses fones ficava ao lado esquerdo do participante, e o outro, ao lado direito do experimentador. Ao lado direito do participante, estava instalada uma filmadora de vídeo. A mesa situava-se em uma sala medindo 4,48 x 2,46 x 3,30 m. A sala era refrigerada por um condicionador de ar.

Os estímulos modelo e de comparação eram peças de madeira, partes de quatro conjuntos iguais de blocos lógicos (marca FUNBEC), variando em três dimensões: forma (quadrado, círculo, retângulo e triângulo), cor (azul, vermelha e amarela) e espessura (grossa e fina). Estas peças de madeira formavam 40 diferentes arranjos de estímulos, cada arranjo constituído de um estímulo-modelo e três estímulos de comparação. Cada estímulo de comparação possuía apenas uma dimensão (cor, espessura ou forma) em comum com o estímulo-modelo e diferia nas demais. Os arranjos de estímulos, previamente preparados, ficavam sobre a mesa, ao lado do experimentador, na ordem em que eram apresentados em cada tentativa.

Para facilitar o manejo dos arranjos, sentado ao lado esquerdo do experimentador, um auxiliar de pesquisa aproximava os arranjos, conforme os mesmos iam sendo apresentados. Os estímulos eram apresentados ao participante através da abertura na base do anteparo divisor da mesa, em uma bandeja de madeira em forma de ' $\mathrm{T}$ '. $\mathrm{Na}$ parte final do cabo dessa bandeja, rente à base retangular, quatro ripas de madeira formavam um quadrado no qual era colocado o estímulo-modelo. Na base retangular, dividida por ripas de madeira em três quadrados, eram apresentados os três estímulos de comparação. As respostas não-verbais (apontar para os estímulos de comparação) emitidas pelos participantes eram registradas pelo experimentador em um protocolo previamente preparado e gravadas pela filmadora, para análises posteriores. Os reforçadores utilizados eram pontos, registrados no contador, que eram trocados por dinheiro ao final do experimento. Cada ponto valia $\mathrm{R} \$ 0,50$ (cinquenta centavos de real).

Durante as sessões experimentais, que duravam em média 30 minutos, participante e experimentador ficavam sentados à mesa, de frente um para o outro, separados pelo anteparo divisor da mesa. A lâmpada na borda superior do anteparo ficava constantemente acesa, voltada para o participante, de maneira a assegurar que seu lado apresentasse iluminação em maior intensidade, garantindo que apenas as ações emitidas pelo participante, bem como o arranjo de estímulos apresentado, pudessem ser observados através do espelho.

\section{Procedimento}

\section{Tentativas}

Em cada tentativa, o experimentador apresentava um dos 40 arranjos de estímulos. Na presença destes estímulos, o participante devia apontar para cada um dos três estímulos de comparação em uma dada sequência. Caso a sequência de respostas emitida estivesse de acordo com as contingências de reforço programadas (sequência correta), um ponto era acrescentado no contador, a lâmpada transparente era acesa e apagada e a bandeja com o arranjo de estímulos era retirada. Caso a sequência de respostas fosse incorreta, a lâmpada transparente não era acesa e a bandeja com o arranjo de estímulos era retirada, sem que fosse acrescentado um ponto no contador. Havia um intervalo variável de aproximadamente 5 segundos entre uma tentativa e outra.

\section{Orientações preliminares}

Na primeira sessão, quando participante e experimentador entravam na sala, a bandeja com um arranjo de estímulos estava sobre a mesa, visível ao participante. O experimentador pedia ao participante que se sentasse na cadeira e solicitava que lesse e assinasse o Termo de Consentimento Livre e Esclarecido. Em seguida, ao lado do participante, sempre apontando com o dedo para cada um dos estímulos a que se referia, dizia:

"Este objeto, aqui em cima, é um modelo. Estes três objetos, aqui em baixo, são para você comparar com o modelo. Nós vamos chamar estes três objetos, aqui em baixo, de objetos de comparação. Observe que cada um destes três objetos de comparação tem uma única propriedade comum ao modelo. [Veja: Este só tem a espessura comum ao modelo. Este aqui só tem a cor comum ao modelo. Este aqui só tem a forma igual ao modelo]. Quando eu apresentar os objetos de comparação para você, você deverá apontar com o dedo para cada um dos três objetos de comparação em uma dada sequência. Durante a pesquisa você poderá ganhar pontos que serão trocados por dinheiro. Cada ponto que você ganhar será trocado por $\mathrm{R} \$ 0,50$ (cinquenta centavos de real), mas apenas no final da pesquisa. Quando você ganhar pontos, os pontos sempre aparecerão 
aqui, neste contador, e esta lâmpada será acesa. Veja como os pontos aparecem no contador e como a lâmpada será acesa (o auxiliar, que se encontrava no outro lado da mesa, acionava o contador e acendia a lâmpada por cinco vezes). Quando você não ganhar pontos, nenhum ponto será acrescentado no contador e a lâmpada não será acesa. Entendeu?"

Este procedimento era repetido por duas vezes e ocorria apenas no início da primeira sessão. Na segunda vez em que estas orientações preliminares eram apresentadas, o trecho entre colchetes era omitido.

\section{Instruções e perguntas}

Logo após as orientações preliminares terem sido apresentadas ao participante, o experimentador solicitava ao participante que colocasse os fones de ouvido; então, o experimentador se deslocava em direção à sua cadeira. Separado do participante pelo anteparo com espelho unidirecional, o experimentador também colocava os fones de ouvido e entregava ao participante, pela abertura na base do anteparo, uma folha de papel contendo uma das seguintes instruções ou perguntas impressas:

Instrução mínima LB: Instrução que não especificava sequência de respostas e que era apresentada na linha de base. [Esta instrução era apresentada no início do experimento para avaliar (durante 10 tentativas, na ausência de consequências diferencias para as sequências de respostas) se o participante mostrava alguma preferência por alguma sequência de respostas, antes da introdução das outras instruções e perguntas].

"Aponte com o dedo para os objetos de comparação".

Instrução correspondente (IC): Instrução que especificava a sequência de respostas que produzia reforço, na sessão em que era apresentada.

"Quando eu mostrar estes objetos para você, você deve fazer o seguinte: Você deve apontar primeiro para a mesma cor [forma ou espessura ${ }^{3}$, depois para a mesma espessura [cor ou forma] e em seguida para a mesma forma [espessura ou cor]."

Instrução mínima (IM). Instrução que não especificava sequência de respostas.

"Quando eu mostrar estes objetos para você, você deve fazer o seguinte:

Você deve apontar para cada um dos três objetos de comparação em sequência para ganhar pontos".

Pergunta correspondente (PC): Pergunta que especificava a sequência de respostas que produzia reforço, na sessão em que era apresentada. (Procurou-se usar uma pergunta correspondente formalmente similar à instrução correspondente para tornar os seus efeitos comparáveis).

"Quando eu mostrar estes objetos para você, o que você deve fazer?

Você deve apontar primeiro para a mesma cor [forma ou espessura $^{4}$ ], depois para a mesma espessura [cor ou forma] e em seguida para a mesma forma [espessura ou cor]?

3 Dependendo da sessão em que a instrução correspondente era apresentada, ela podia especificar uma das seguintes sequências de respostas: Cor (C) - Espessura (E) - Forma (F), FCE, ECF, EFC ou CFE.

4 Dependendo da sessão em que a pergunta correspondente era apresentada, ela especificava uma das seguintes seqüências de respostas: $\mathrm{CEF}$, FCE, ECF, EFC ou CFE.
Pergunta mínima (PM): Pergunta que não especificava a sequência de respostas que produzia reforço. (Procurou-se usar uma pergunta mínima formalmente similar à instrução mínima para tornar os seus eventuais efeitos comparáveis).

"Quando eu mostrar estes objetos para você, o que você deve fazer?

Você deve apontar para cada um dos três objetos de comparação em sequência para ganhar pontos?"

\section{Forma de apresentação das instruções e perguntas}

Imediatamente após o participante ter recebido a folha de papel contendo uma das instruções ou perguntas impressa, o experimentador ligava o tape-deck e, por intermédio dos fones de ouvido, o participante ouvia uma fita, previamente gravada, que dizia:

"Eu vou ler (dependendo da fase experimental, a gravação dizia: "esta instrução" ou "esta pergunta") para você em voz alta. Acompanhe minha leitura, lendo silenciosamente". A gravação continuava com a leitura da instrução ou da pergunta contida na folha de papel. Terminada essa primeira leitura, a gravação prosseguia: "Agora, você deve ler (dependendo da fase experimental, a gravação dizia: "esta instrução" ou "esta pergunta") sozinho, silenciosamente. Leia com calma e bastante atenção. Você tem todo o tempo que achar necessário para entendê-la. Quando você achar que entendeu bem (dependendo da fase experimental, a gravação dizia: "a instrução" ou "a pergunta"), avise-me". Terminada esta segunda leitura, a gravação prosseguia: "Devolva-me a folha com (dependendo da fase experimental, a gravação dizia: "a instrução" ou "a pergunta"). Eu só posso falar com você agora, no início da próxima sessão. Você pode retirar os fones de ouvido agora."

Esse procedimento era usado no início de cada fase, nas oito condições experimentais. Logo após o participante ter devolvido a folha, o experimentador removia a bandeja, tornava a apresentar a bandeja com um novo arranjo de estímulos, e dizia: "Comece a apontar".

\section{Delineamento experimental}

Os participantes foram distribuídos em oito condições experimentais, como indicado na Tabela 1. Cada condição, realizada com três participantes, consistia de cinco fases. A Fase 1 (linha de base) de cada condição era iniciada com a apresentação da instrução mínima LB. As condições diferiam apenas quanto à ordem em que as instruções (correspondente e mínima) e as perguntas (correspondente e mínima) eram apresentadas no início da primeira sessão de cada uma das Fases 2, 3, 4 e 5 .

Assim, na Condição 1 (IC-IM-PC-PM), a Fase 2 era iniciada com a apresentação da instrução correspondente (IC); a Fase 3, com a instrução mínima (IM); a Fase 4, com a pergunta correspondente (PC); e a Fase 5, com a pergunta mínima (PM). Na Condição 2 (PC-PM-IC-IM), a Fase 2 era iniciada com a pergunta correspondente (PC); a Fase 3, com a pergunta mínima (PM); a Fase 4, com a instrução correspondente (IC); e, a Fase 5, com a instrução mínima (IM). Na Condição 3 (IM-IC-PM-PC), a instrução mínima, 
a instrução correspondente, a pergunta mínima e a pergunta correspondente eram apresentadas no início da primeira sessão das Fases 2, 3, 4 e 5, respectivamente. Já na Condição 4 (PM-PC-IM-IC), a ordem de apresentação das instruções e perguntas no início da primeira sessão das Fases 2, 3, 4 e 5 era: pergunta mínima, pergunta correspondente, instrução mínima e instrução correspondente, respectivamente. Na Condição 5 (IC-PM-PC-IM), a ordem de apresentação era: instrução correspondente, pergunta mínima, pergunta correspondente e instrução mínima. Na Condição 6 (PC-IM-IC-PM), a ordem de apresentação era: pergunta correspondente, instrução mínima, instrução correspondente e pergunta mínima. Na Condição 7 (IM-PC-PM-IC), a ordem de apresentação era: instrução mínima, pergunta correspondente, pergunta mínima e instrução correspondente. E na Condição 8 (PM-IC-IM-PC), a ordem de apresentação era: pergunta mínima, instrução correspondente, instrução mínima e pergunta correspondente.

Em cada uma das oito condições, a Fase 1 era encerrada após a ocorrência de 10 tentativas e cada uma das demais fases era encerrada após duas sessões terem sido completadas. A partir da Fase 2, inclusive, cada sessão era encerrada obedecendo a um dos seguintes critérios, o que ocorresse primeiro: (a) após a obtenção de 10 pontos consecutivos ou, (b) após a ocorrência de 80 tentativas.
Durante a Fase 1, nenhuma sequência de respostas era reforçada. Durante a primeira sessão da Fase 2, era reforçada apenas uma de duas sequências de repostas: (a) a sequência de respostas Cor-Espessura-Forma (CEF) ou (b) a sequência de respostas Forma-Cor-Espessura. Qual destas duas sequências de respostas seria reforçada, dependia do desempenho do participante na Fase 1. Se na Fase 1 o participante tivesse emitido a sequência de respostas CEF em mais de 50\% das tentativas, a sequência de respostas reforçada no início da Fase 2 seria FCE. Caso contrário, a sequência de respostas reforçada no início da Fase 2 seria CEF.

A transição da primeira para a segunda sessão da Fase 2, bem como a transição da primeira para a segunda sessão de cada uma das demais fases subsequentes, era marcada pela mudança não sinalizada nas contingências de reforço programadas. Este procedimento foi usado para testar, dentro de cada fase, a sensibilidade do comportamento à mudança nas contingências programadas. Assim, na segunda sessão da Fase 2, a única sequência de respostas reforçada era EFC. Durante a Fase 3, a sequência de respostas reforçada na primeira sessão era ECF e na segunda sessão era CFE. Durante a Fase 4, a sequência de respostas reforçada na primeira sessão era EFC e na segunda sessão era CEF. Durante a Fase 5, a sequência de respostas reforçada na primeira sessão era CFE e na segunda sessão era FCE. Em cada uma das Fases 2, 3,

Tabela 1. Esquema do procedimento.

\begin{tabular}{|c|c|c|c|c|c|c|c|c|}
\hline \multirow{7}{*}{ Condições } & \multicolumn{2}{|c|}{ Fase 2} & \multicolumn{2}{|c|}{ Fase 3} & \multicolumn{2}{|c|}{ Fase 4} & \multicolumn{2}{|c|}{ Fase 5} \\
\hline & Sessão 1 & Sessão 2 & Sessão 1 & Sessão 2 & Sessão 1 & Sessão 2 & Sessão 1 & Sessão 2 \\
\hline & \multicolumn{8}{|c|}{ Seqüências reforçadas } \\
\hline & $\mathrm{CEF}$ & & & & & & & \\
\hline & ou & $\mathrm{EFC}$ & ECF & CFE & $\mathrm{EFC}$ & $\mathrm{CEF}$ & CFE & FCE \\
\hline & $\mathrm{FCE}$ & & & & & & & \\
\hline & \multicolumn{8}{|c|}{ Ordem de apresentação dos estímulos verbais na Sessão 1} \\
\hline 1 & IC & & IM & & $\mathrm{PC}$ & & PM & \\
\hline 2 & $\mathrm{PC}$ & & PM & & IC & & IM & \\
\hline 3 & $\mathrm{IM}$ & & IC & & PM & & $\mathrm{PC}$ & \\
\hline 4 & $\mathrm{PM}$ & & $\mathrm{PC}$ & & IM & & IC & \\
\hline 5 & IC & & PM & & PC & & IM & \\
\hline 6 & $\mathrm{PC}$ & & IM & & IC & & PM & \\
\hline 7 & IM & & $\mathrm{PC}$ & & PM & & $\mathrm{IC}$ & \\
\hline 8 & PM & & IC & & IM & & PC & \\
\hline
\end{tabular}

Nota: Cada condição foi realizada com três participantes. $\mathrm{CEF}=$ Sequência de respostas cor, espessura, forma; $\mathrm{FCE}=\mathrm{Sequência}$ forma, cor, espessura; $\mathrm{EFC}=$ Sequência espessura, cor, forma $\mathrm{ECF}=$ Sequência espessura, cor, forma; $\mathrm{CFE}=$ Sequência cor, forma, espessura; $\mathrm{IC}=$ Instrução correspondente; IM = Instrução mínima; $\mathrm{PC}=$ Pergunta correspondente; PM = Pergunta mínima. 
4 e 5 , a sequência de repostas correta era reforçada em um esquema de reforço razão fixa 6 (FR6).

\section{Comparação dos registros (acordo entre observadores)}

Nas oito condições, a cada duas sessões, um observador independente comparava o registro feito pelo experimentador com o registro feito pela filmadora. Caso houvesse $100 \%$ de concordância entre os registros, o participante continuava participando do experimento. Caso contrário, a sua participação no experimento era encerrada. Não ocorreram erros de concordância entre os registros durante o experimento.

\section{Término da participação do estudante no experimento}

Antes de o início de cada sessão (isto é, antes de o participante entrar na sala experimental), o contador era zerado. Portanto, os pontos eram registrados cumulativamente, no contador, apenas dentro de uma mesma sessão. No entanto, a partir da segunda sessão da Fase 2, logo após o participante ter entrado na sala experimental, e sempre antes de o início de cada sessão subsequente, o participante era informado pelo experimentador sobre o número total de pontos obtidos nas sessões anteriores.

O início e o encerramento de uma sessão eram marcados, respectivamente, pela entrada e saída do participante da sala experimental. As sessões ocorriam diariamente, sendo realizadas duas sessões por dia, no máximo. O intervalo entre as sessões em um mesmo dia era de 10 minutos, aproximadamente. A participação no experimento era encerrada quando o participante atingia o critério de encerramento da Fase 5, quando era feito o pagamento dos pontos obtidos pelo participante.

\section{Resultados}

Serão apresentados os dados de cada fase. A partir da Fase 2, inclusive, serão apresentados os dados da primeira sessão e depois os dados da segunda sessão de cada fase. Na primeira sessão, serão descritos os dados que mostram se os estímulos antecedentes verbais (primeiro a instrução correspondente, depois a pergunta correspondente e, finalmente, a pergunta e a instrução mínima) estabeleceram, ou não, o comportamento correto ${ }^{5}$. Na segunda sessão, serão descritos os dados que mostram se o comportamento muda, ou não, acompanhando a mudança nas contingências, isto é, se o comportamento apresenta ou não sensibilidade às contingências. Após a descrição da Fase 1, a Fase 2 será descrita com detalhes. Em seguida será apresentada uma síntese dos resultados.

Durante a Fase 1 (linha de base), os Participantes P22, da Condição 2, e P42, da Condição 4, foram os únicos que apresentaram a sequência de respostas CEF em mais de $50 \%$ das tentativas. Portanto, para estes dois participantes a

5 O comportamento correto era a emissão de uma determinada sequência específica, programada para produzir reforço em cada sessão. sequência correta no início da Fase 2 foi FCE. Para os demais participantes a sequência correta no início da Fase 2 foi CEF.

A Figura 1 mostra a frequência acumulada de sequências de respostas, emitidas durante as Fases 2, 3, 4 e 5, pelos Participantes P11, P12 e P13, da Condição 1 (IC-IM-PC-PM); P21, P22 e P23, da Condição 2 (PC-PM-IC-IM); P31, P32 e P33, da Condição 3 (IM-IC-PM-PC); P41, P42 e P43, da Condição 4 (PM-PC-IM-IC); P51, P52 e P53, da Condição 5 (IC-PM-PC-IM); P61, P62 e P63, da Condição 6 (PC-IM-IC-PM); P71, P72 e P73, da Condição 7 (IM-PC-PM-IC); e, P81, P82 e P83, da Condição 8 (PM-IC-IM-PC).

Pode-se notar, na primeira sessão da Fase 2, quando a instrução correspondente às contingências foi apresentada pela primeira vez nas Condições 1 e 5, que cinco (P11, P12, P13, P52 e P53) dos seis participantes responderam corretamente, de acordo com essa instrução, em todas as tentativas dessa sessão. P51 apresentou sequências incorretas.

Em contraste, quando a pergunta correspondente foi apresentada pela primeira vez, na primeira sessão da Fase 2 das Condições 2 e 6, somente dois (P21 e P23) dos seis participantes responderam corretamente em todas as tentativas dessa sessão. P22 emitiu uma sequência (CEF) incorreta. P61, P62 e P63 apresentaram um desempenho variável, isto é, emitiram, ora uma ora outra, as sequências CEF, CFE, EFC, ECF, FCE e FEC.

Também diferente dos participantes que foram inicialmente expostos à instrução correspondente, quando a instrução mínima (na primeira sessão da Fase 2 das Condições 3 e 7) e a pergunta mínima (na primeira sessão da Fase 2 das Condições 4 e 8 ) foram apresentadas pela primeira vez, apenas um (P82) dos 12 participantes respondeu corretamente durante a maior parte da primeira sessão.

Quatro participantes (P33, P71, P72 e P81) responderam predominantemente em uma única sequência incorreta. P33 emitiu a sequência FCE. P71 emitiu a sequência CFE. P72 emitiu a sequência ECF. E P81 emitiu a sequência FCE. Os outros sete participantes restantes (P31, P32, P41, P42, P43, P73 e P83) apresentaram um desempenho variável, semelhante ao apresentado por P61, P62 e P63.

Na Figura 1 também se pode notar, na segunda sessão da Fase 2, quando as contingências foram alteradas sem sinalização, que nenhum dos 24 participantes respondeu corretamente durante toda a sessão. Dos cinco participantes que haviam seguido a instrução correspondente na primeira sessão da Fase 2, quatro (P11, P12, P13 e P53) apresentaram um desempenho insensível ${ }^{6}$ à mudança nas contingências na segunda sessão dessa fase, isto é, continuaram seguindo a instrução, apesar de o seguimento de instrução não mais

6 "O termo insensibilidade está sendo usado para descrever o comportamento que não está sob o controle de suas consequências imediatas e o termo sensibilidade está sendo usado para descrever o comportamento que está sob o controle de suas consequências imediatas, em uma determinada situação particular (Albuquerque \& cols., 2003). Por essa definição, um critério para avaliar a sensibilidade ou a insensibilidade do comportamento é verificar se o comportamento ocorre sob controle de suas consequências imediatas ou se ocorre independentemente de tais consequências. Isso pode ser feito pelo menos de duas maneiras: 1) mantendo inalteradas as contingências de reforço programadas no experimento e manipulando as regras; e, 2) mantendo inalteradas as regras e manipulando as contingências de reforço programadas no experimento" (Albuquerque \& cols., 2008; p. 327). 

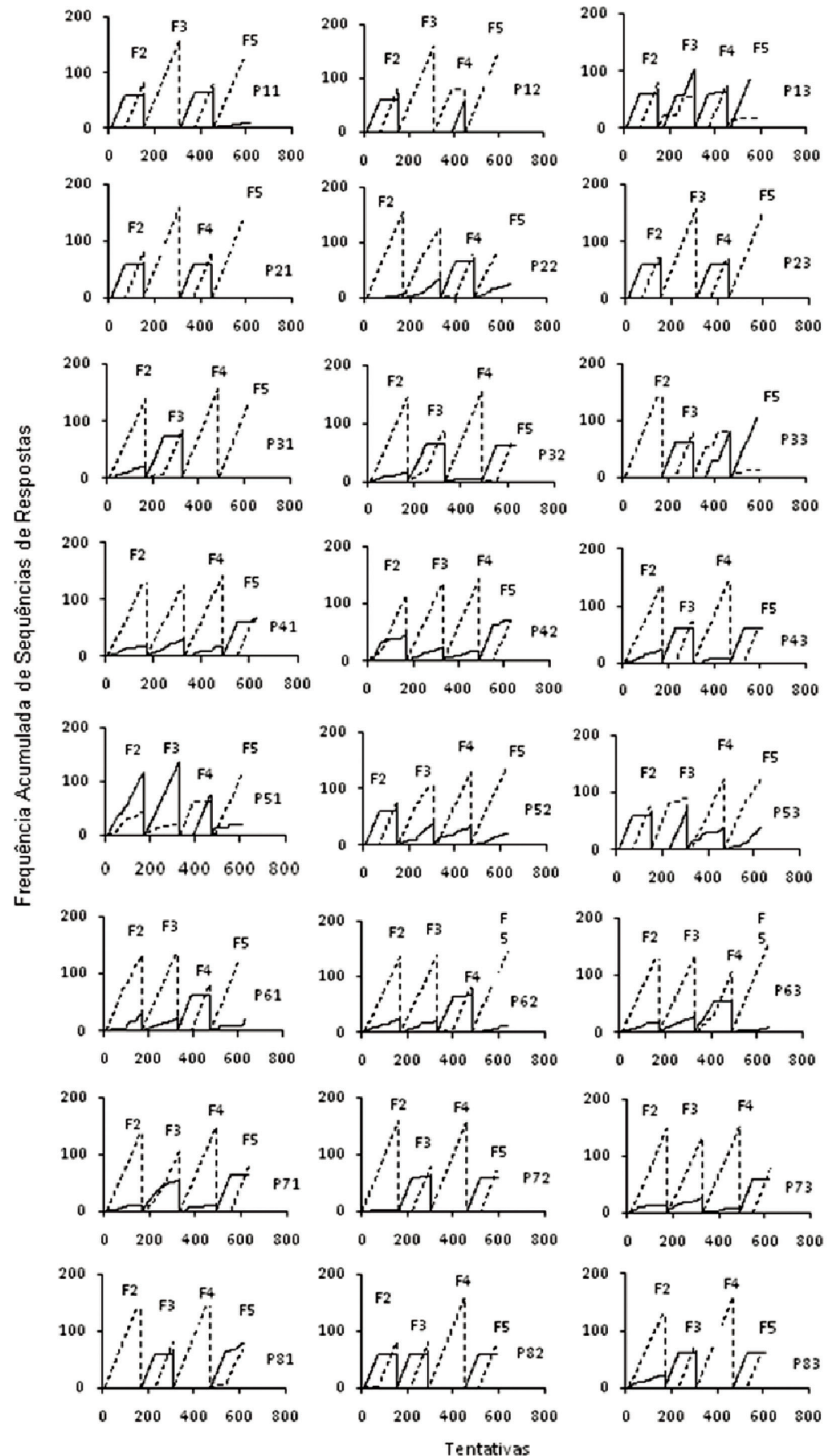

Figura 1. Frequência acumulada de sequências corretas (linha sólida) e incorretas (linha tracejada), para cada participante (P), nas Fases (F) 2, 3, 4 e 5 das 8 condições experimentais. Quebra na curva acumulada indica mudança de fase. 
produzir ponto. Dos dois participantes que haviam respondido de acordo com a pergunta correspondente na primeira sessão da Fase 2, um (P21) continuou apresentando esse mesmo comportamento na segunda sessão dessa fase, ou seja, apresentou um desempenho insensível à mudança nas contingências. P82 (da Condição 8 - iniciada com a instrução mínima) também apresentou um desempenho insensível à mudança nas contingências. Quanto aos demais participantes, 11 (P31, P32, P33, P41, P42, P43, P51, P61, P62, P63 e P83) apresentaram um desempenho variável; três (P71 P72 e P81) responderam de acordo com as suas histórias experimentais, isto é, continuaram emitindo a mesma sequência emitida na sessão anterior; um (P73) respondeu incorretamente em uma única sequência; e, um (P52) abandonou o seguimento da instrução correspondente.

Em síntese, durante a maior parte da primeira sessão das fases iniciadas com a apresentação da instrução correspondente (Fase 2 das Condições 1 e 5, Fase 3 das Condições 3 e 8 , Fase 4 das Condições 2 e 6 e Fase 5 das Condições 4 e 7), 23 dos 24 participantes responderam corretamente, de acordo com a instrução correspondente às contingências. Desses 23, 22 participantes continuaram seguindo a instrução correspondente, após a mudança nas contingências de reforço, durante a maior parte da segunda sessão dessas fases. P51 foi o único que não seguiu a instrução correspondente durante a primeira sessão.

Durante a maior parte da primeira sessão das fases iniciadas com a apresentação da pergunta correspondente (Fase 2 das Condições 2 e 6, Fase 3 das Condições 4 e 7, Fase 4 das Condições 1 e 5 e Fase 5 das Condições 3 e 8), 12 (P11, P13, P21, P23, P32, P33, P43, P51, P72, P81, P82 e P83) dos 24 participantes responderam corretamente, de acordo com a pergunta correspondente. Desses 12, oito (P11, P21, P32, P43, P72, P81, P82 e P83) apresentaram um desempenho insensível à mudança nas contingências de reforço durante a maior parte da segunda sessão dessas fases. Os demais participantes apresentaram um de três padrões de comportamentos. Nove participantes (P41, P42, P52, P53, P61, P62, $\mathrm{P} 63,71$ e P73) apresentaram um desempenho variável, dois (P22 e P31) responderam predominantemente em uma única sequência incorreta e um (P12) respondeu de acordo com sua história experimental.

$\mathrm{Na}$ primeira sessão das fases iniciadas com a apresentação da instrução mínima (Fase 2 das Condições 3 e 7, Fase 3 das Condições 1 e 6, Fase 4 das Condições 4 e 8 e Fase 5 das Condições 2 e 5), apenas 2 (P13 e P51) dos 24 participantes responderam corretamente, de acordo com as contingências programadas. Na segunda sessão dessas fases, P13 apresentou um desempenho variável e P51 respondeu predominantemente na sequência incorreta FCE. Os demais participantes apresentaram um de três padrões de comportamentos. Dez participantes (P22, P31, P32, P41, P42, P53, P61, P62, P63 e P73) apresentaram um desempenho variável, seis (P23, P33, P52, P71, P72 e P82) responderam predominantemente em uma única sequência incorreta e 6 (P11, P12, P21, P43, P81 e P83) responderam de acordo com suas histórias experimentais.

Na primeira sessão das fases iniciadas com a apresentação da pergunta mínima (Fase 2 das Condições 4 e 8, Fase 3 das Condições 2 e 5, Fase 4 das Condições 3 e 7, e Fase 5 das
Condições 1 e 6), apenas 2 (P13 e P82) dos 24 participantes responderam corretamente, de acordo com as contingências programadas. Na segunda sessão dessas fases, P13 apresentou um desempenho sensível e P82 continuou emitindo a mesma sequência emitida na sessão anterior, isto é, apresentou um desempenho insensível à mudança nas contingências de reforço. Os demais participantes apresentaram um de três padrões de comportamentos. Dez participantes (P22, P23, P33, P41, P42, P43, P51, P52, P63 e P83) apresentaram um desempenho variável, sete (P11, P12, P53, P71, P72, P73 e $\mathrm{P} 81)$ responderam predominantemente em uma única sequência incorreta e cinco (P21, P31, P32, P61 e P62) responderam de acordo com suas histórias experimentais.

Finalmente, a Tabela 2 mostra outra diferença entre o controle pela instrução e pela pergunta correspondente. Pode-se notar que a instrução correspondente, quando comparada com a pergunta correspondente, gerou menor variabilidade do comportamento em relação à regra. Ou seja, 20 dos 24 participantes seguiram a instrução correspondente em $100 \%$ das 12 primeiras tentativas da primeira sessão das fases iniciadas com a apresentação dessa instrução. Também 20 dos 24 participantes seguiram a instrução correspondente (isto é, emitiram a sequência previamente especificada por essa instrução) em $100 \%$ das 12 primeiras tentativas da segunda sessão (quando era testada a sensibilidade do comportamento à mudança não sinalizada nas contingências) das fases iniciadas com a apresentação dessa instrução. Diferentemente, apenas 9 dos 24 participantes seguiram a pergunta correspondente em 100\% das 12 primeiras tentativas da primeira sessão das fases iniciadas com a apresentação dessa pergunta. Também apenas 8 dos 24 participantes seguiram a pergunta correspondente em $100 \%$ das 12 primeiras tentativas da segunda sessão das fases iniciadas com a apresentação dessa instrução.

\section{Discussão}

O presente estudo procurou verificar que características um estímulo antecedente verbal deve apresentar para ele poder exercer a função de regras de estabelecer comportamentos novos independentemente de suas consequências imediatas.

Os resultados mostraram que a instrução correspondente às contingências estabeleceu o comportamento considerado correto na primeira sessão das fases em que foi apresentada e gerou insensibilidade desse comportamento à mudança nas contingências programadas na segunda sessão dessas fases em 23 dos 24 casos possíveis (correspondente a 95\%). De modo similar, a pergunta correspondente às contingências também chegou a exercer aquela função de regras, mas isso ocorreu apenas em 8 dos 24 casos possíveis (correspondente a 33\%). Em contraste, nas sessões iniciadas com a instrução mínima apenas 2 dos 24 participantes responderam corretamente, e nas sessões iniciadas com a pergunta mínima, também apenas 2 dos 24 participantes responderam corretamente.

Tais diferenças de resultados ocorreram, em parte, devido às diferenças nas características formais dos estímulos antecedentes verbais apresentados aos participantes. Ou seja, a instrução e a pergunta correspondente mostraram maior eficiência em estabelecer o comportamento correto e 
Tabela 2. Porcentagens de seguimento da instrução correspondente (IC) e da pergunta correspondente (PC) nas primeiras 12 tentativas da primeira ( $\left.1^{\text {a }} \mathrm{S}\right)$ e da segunda ( $\left(2^{\mathrm{a}} \mathrm{S}\right)$ sessão de cada uma das fases iniciadas com a apresentação desses estímulos.

\begin{tabular}{|c|c|c|c|c|c|c|c|c|c|c|c|c|c|c|c|c|c|}
\hline \multirow{3}{*}{ Condições } & \multirow{3}{*}{ Participantes } & \multicolumn{4}{|c|}{ Fase 2} & \multicolumn{4}{|c|}{ Fase 3} & \multicolumn{4}{|c|}{ Fase 4} & \multicolumn{4}{|c|}{ Fase 5} \\
\hline & & \multirow{2}{*}{$\begin{array}{r}\text { IC } \\
1^{a} \mathrm{~S}\end{array}$} & \multicolumn{3}{|c|}{$\mathrm{PC}$} & \multicolumn{2}{|l|}{ IC } & \multicolumn{2}{|l|}{$\mathrm{PC}$} & \multirow{2}{*}{$\begin{array}{r}\text { IC } \\
1^{\text {a }} \mathrm{S}\end{array}$} & \multicolumn{3}{|c|}{$\mathrm{PC}$} & \multirow{2}{*}{$\frac{\mathrm{IC}}{1^{\mathrm{a}} \mathrm{S}}$} & \multicolumn{3}{|c|}{$\mathrm{PC}$} \\
\hline & & & $2^{a} S$ & $1^{a} \mathrm{~S}$ & $2^{a} S$ & $1^{a} \mathrm{~S}$ & $2^{a} S$ & $1^{\text {a } S}$ & $2^{a} S$ & & $2^{a} S$ & $1^{a} \mathrm{~S}$ & $2^{a} S$ & & $2^{a} S$ & $1^{a} S$ & $2^{\mathrm{a}} \mathrm{S}$ \\
\hline \multirow[t]{3}{*}{1} & P11 & 100 & 100 & & & & & & & & & 92 & 92 & & & & \\
\hline & $\mathrm{P} 12$ & 100 & 100 & & & & & & & & & 0 & 0 & & & & \\
\hline & P13 & 100 & 100 & & & & & & & & & 100 & 67 & & & & \\
\hline \multirow[t]{3}{*}{2} & P21 & & & 100 & 100 & & & & & 100 & 100 & & & & & & \\
\hline & $\mathrm{P} 22$ & & & 8 & 25 & & & & & 75 & 100 & & & & & & \\
\hline & $\mathrm{P} 23$ & & & 100 & 100 & & & & & 100 & 92 & & & & & & \\
\hline \multirow[t]{3}{*}{3} & P31 & & & & & 100 & 100 & & & & & & & & & 8 & 0 \\
\hline & P32 & & & & & 83 & 100 & & & & & & & & & 100 & 100 \\
\hline & P33 & & & & & 100 & 100 & & & & & & & & & 42 & 0 \\
\hline \multirow[t]{3}{*}{4} & P41 & & & & & & & 25 & 3 & & & & & 100 & 100 & & \\
\hline & P42 & & & & & & & 8 & 8 & & & & & 100 & 100 & & \\
\hline & $\mathrm{P} 43$ & & & & & & & 100 & 100 & & & & & 100 & 100 & & \\
\hline \multirow[t]{3}{*}{5} & P51 & 25 & 33 & & & & & & & & & 100 & 58 & & & & \\
\hline & P52 & 100 & 100 & & & & & & & & & 75 & 17 & & & & \\
\hline & P53 & 100 & 83 & & & & & & & & & 67 & 8 & & & & \\
\hline \multirow[t]{3}{*}{6} & P61 & & & 0 & 0 & & & & & 100 & 100 & & & & & & \\
\hline & P62 & & & 17 & 17 & & & & & 100 & 100 & & & & & & \\
\hline & $\mathrm{P} 63$ & & & 17 & 0 & & & & & 92 & 75 & & & & & & \\
\hline \multirow[t]{3}{*}{7} & P71 & & & & & & & 83 & 33 & & & & & 100 & 100 & & \\
\hline & P72 & & & & & & & 100 & 100 & & & & & 100 & 100 & & \\
\hline & P73 & & & & & & & 58 & 25 & & & & & 100 & 100 & & \\
\hline \multirow[t]{3}{*}{8} & P81 & & & & & 100 & 100 & & & & & & & & & 75 & 100 \\
\hline & P82 & & & & & 100 & 100 & & & & & & & & & 100 & 100 \\
\hline & P83 & & & & & 100 & 100 & & & & & & & & & 100 & 100 \\
\hline
\end{tabular}

em gerar insensibilidade desse comportamento à mudança nas contingências programadas, quando comparadas com a instrução e a pergunta mínima, em parte, porque a instrução e a pergunta correspondente especificavam o comportamento correto, enquanto que a instrução e a pergunta mínima não especificavam tal comportamento. Por essa proposição, então, para um estímulo antecedente verbal poder exercer a função de estabelecer um comportamento é necessário que ele especifique o comportamento a ser estabelecido.

Contudo, especificar o comportamento a ser estabelecido não é uma condição suficiente para que um estímulo antecedente verbal possa estabelecer esse comportamento (Albuquerque \& Ferreira, 2001; Braga \& cols., 2005). Os dados mostrando que a instrução correspondente funcionou como regra em $95 \%$ dos casos possíveis, enquanto que a pergunta correspondente funcionou da mesma maneira em apenas $33 \%$ dos casos possíveis, apóiam essa sugestão. Estes dados replicam os dados do estudo de Braga e cols. e sugerem que a instrução correspondente mostrou maior eficiência em estabelecer o comportamento correto e em gerar insensibilidade desse comportamento à mudança nas contingências programadas, quando comparada com a pergunta correspondente, em parte, porque a instrução correspondente afirmava que o comportamento especificado deveria ser emitido, enquanto que a pergunta correspondente questionava (isto é, colocava em dúvida) se o comportamento especificado deveria ser emitido. Em síntese, estes dados sugerem que um estímulo antecedente verbal tem maior probabilidade de estabelecer um comportamento quando a emissão do comportamento que ele especifica é afirmada do que quando a emissão de tal comportamento é questionada.

Além das características formais dos estímulos antecedentes verbais, outras variáveis podem ter contribuído para determinar o desempenho final apresentado pelos participantes. Pode-se dizer que a instrução e a pergunta correspondente estabeleceram o comportamento por elas especificado, nos casos previamente descritos na seção de resultados, por quatro razões: 1) antes de o estímulo verbal (a instrução ou a pergunta correspondente) ser apresentado, a sequência correta não estava sendo emitida; 2) depois de o estímulo verbal ser apresentado, a sequência correta passou a ser emitida; 3 ) a sequência correta emitida correspondia à especificada pelo estímulo verbal; e, 4) a sequência correta foi emitida antes mesmo que as consequências programadas pudessem ter exercido algum efeito sobre ela, já que apenas a emissão consecutiva da sexta sequência correta produzia o reforço programado. Depois de estabelecida, a sequência correta tornava-se incorreta quando ela continuava sendo mantida após a mudança nas contingências. A manutenção dessa sequência, apesar de não mais produzir o reforço programado, era uma evidência adicional de que a sua emissão estava sob controle antecedente do estímulo verbal. Utilizou-se o termo insensibilidade do seguimento de regra à mudança nas contingências para descrever os casos em que a sequência, sob controle antecedente de regra, foi mantida após a mudança nas contingências. 
Essa insensibilidade pode ter ocorrido, em parte, devido: 1) à presença do experimentador na sessão experimental, uma vez que ele pode ter funcionado como um estímulo na presença do qual o seguimento de regra produziria aprovação social e o não seguimento de regra produziria desaprovação social (Albuquerque \& cols., 2004; Barret \& cols., 1987; Cerutti, 1994; Hayes \& cols., 1986; Malott, 1989; Torgrud \& Holborn, 1990); e, 2) ao esquema de reforço intermitente utilizado, uma vez que este esquema poderia ter dificultado a discriminação da discrepância entre as consequências especificadas na regra e as consequências produzidas pelo seguimento de regras e, deste modo, facilitado à insensibilidade do seguimento de regra à mudança nas contingências (Baron \& Galizio, 1983; Cerutti, 1989; 1991; Joyce \& Chase, 1990; Newman \& cols., 1995).

Admitindo a possibilidade de a presença do experimentador na sessão experimental ter favorecido a manutenção do comportamento sob controle antecedente do estímulo verbal, pode-se supor que este eventual efeito, se ocorreu, foi maior nas fases iniciadas com a instrução correspondente do que nas fases iniciadas com a pergunta correspondente. Isto considerando que nas fases iniciadas com a instrução correspondente, o experimentador claramente monitorava o seguimento de regra, uma vez que ele especificava qual era o comportamento correto e mandava que este comportamento fosse emitido. Diferentemente, nas fases iniciadas com a pergunta correspondente, o experimentador não monitorava claramente o seguimento de regras, já que ele questionava se o participante deveria emitir o comportamento especificado.

Em relação ao esquema de reforço, ele também pode ter contribuído, junto com as propriedades formais dos estímulos verbais, para a ocorrência da variabilidade no desempenho dos participantes, observada em grande parte das sessões iniciadas com a pergunta correspondente, com a instrução mínima e com a pergunta mínima. Ou seja, essa variabilidade pode ter ocorrido devido ao fato de: 1) nas sessões iniciadas com a instrução e com a pergunta mínima o comportamento correto não ter sido especificado; 2) nas sessões iniciadas com a pergunta correspondente o comportamento correto ter sido questionado; e ainda, 3) devido ao fato de a sequência correta não ter sido reforçada imediatamente após a sua emissão, uma vez que ela era reforçada a cada seis emissões consecutivas de uma mesma sequência correta.

Assim, em algumas das sessões iniciadas com a pergunta correspondente, com a instrução mínima e com a pergunta mínima, as contingências não-sociais (representadas aqui pelo esquema de reforço utilizado) contribuíram para determinar a forma do desempenho apresentado pelos participantes. Já nas sessões iniciadas com a instrução correspondente, as contingências sociais (representadas aqui pela presença do experimentador na sessão experimental) podem ter contribuído para a manutenção do comportamento sob controle antecedente de estímulos antecedentes verbais, mas não determinaram a forma inicial deste comportamento. Neste caso, a instrução correspondente determinou a forma inicial do desempenho apresentado pelos participantes. Uma evidência disso é que independentemente das contingências programadas, 23 dos 24 participantes responderam, de acordo com a instrução correspondente nas primeiras tentativas nas sessões iniciadas com essa instrução. Isto ocorreu indepen- dentemente da história experimental do participante, isto é, independentemente da fase em que a instrução correspondente foi apresentada, como pode ser observado na Tabela 2.

Esta análise dos resultados do presente estudo sugere que os efeitos de estímulos antecedentes verbais sobre o comportamento dependem, em parte, das propriedades formais destes estímulos. Mais especificamente, os resultados mostraram que a eficácia de um estímulo antecedente verbal em funcionar como regras, estabelecendo um comportamento novo, depende, em parte, de se o estímulo antecedente verbal especifica ou não o comportamento a ser estabelecido e de se este estímulo questiona ou afirma que o comportamento especificado deve ser emitido. Os estímulos antecedentes verbais que especificam o comportamento a ser estabelecido, diferente dos estímulos que não especificam tal comportamento, tendem a exercer a função de regras de estabelecer um comportamento novo. Os estímulos antecedentes verbais que afirmam (ou mandam) que o comportamento por eles especificado deve ser emitido, quando comparados com os estímulos que questionam se o comportamento por eles especificado deve ser emitido, tendem a exercer aquela função de regras com maior eficiência, na medida em que produzem menor variabilidade do comportamento em relação à regra.

Por esta análise, se apenas os estímulos antecedentes verbais que podem exercer a função de regras de estabelecer um comportamento novo deveriam ser classificados como regras, então, regras não deveriam ser definidas como estímulos antecedentes verbais, como tem sido proposto (Hayes, 1986; Zettle \& Hayes, 1982), uma vez que nem todos os estímulos antecedentes verbais podem exercer essa função de regras. Uma alternativa seria adotar a definição que sugere que regras são estímulos especificadores de contingências (Skinner, 1969). Um problema é que, enquanto a definição de regras como estímulos antecedentes verbais é muito abrangente, uma vez que inclui estímulos que não exercem a principal função de regras, a definição de regras como estímulos especificadores de contingências é muito restritiva, uma vez que exclui estímulos que não especificam claramente contingências. As implicações de se excluir da análise os estímulos que não especificam claramente contingências serão consideradas a seguir.

Até o momento foi enfatizada a visão de que um estímulo antecedente verbal pode ser classificado como regra quando ele pode exercer a função de estabelecer um comportamento novo independentemente de suas consequências imediatas (Albuquerque, 2005). Um problema, no entanto, é que regras não exercem apenas a função de estabelecer um comportamento novo, uma vez que regras também podem alterar as funções de outros estímulos (Schlinger \& Blakely, 1987). Uma implicação de se considerar essa outra função de regras é que os estímulos antecedentes verbais que não especificam o comportamento a ser estabelecido, apesar de não exercerem a função de estabelecer comportamentos novos, poderiam ser classificados como regras, porque poderiam exercer a função de alterar as funções de outros estímulos.

Mas neste caso as características formais dos estímulos verbais também são importantes e dois aspectos devem ser considerados. O primeiro é que quando um estímulo antecedente verbal especifica o comportamento a ser estabelecido, ele pode ser considerado como regra, tanto porque 
pode estabelecer um comportamento novo, quanto porque pode alterar as funções de outros estímulos. Já quando um estímulo antecedente verbal não especifica o comportamento a ser estabelecido, ele só pode ser considerado como regra porque pode alterar as funções de outros estímulos, uma vez que ele não pode estabelecer um comportamento novo. O segundo aspecto é que o primeiro requisito para se dizer que um comportamento está sob controle de regra é que a forma inicial desse comportamento seja estabelecida por uma regra (o segundo requisito, como já dito, é que o seguimento de regra ocorra independentemente de suas consequências imediatas). Se a forma inicial do comportamento é estabelecida por suas consequências ou por observação ou por outra variável qualquer, este comportamento não é puramente controlado por regras (Albuquerque, 2001).

Isto implica que quando um estímulo antecedente verbal especifica o comportamento a ser estabelecido, pode-se dizer que o comportamento que se segue a apresentação desse estímulo está sob controle de regra, desde que esse estímulo tenha estabelecido a forma inicial do comportamento e esse comportamento ocorra independentemente de suas consequências imediatas, como ocorreu na Fase 2 do Participante P11, por exemplo. Mas quando um estímulo antecedente verbal não especifica o comportamento a ser estabelecido, embora ele possa ser considerado como regra nas situações em que altera a função de outros estímulos, não se pode dizer que o comportamento que se segue a apresentação desse estímulo está puramente sob controle de regra, porque a forma desse comportamento não foi determinada pela regra.

Por exemplo, o estímulo antecedente verbal: "Que horas são?" pode contribuir para estabelecer o comportamento de observar um relógio, observar a posição do sol, etc, mas não estabelece o comportamento especifico de dizer 3, 4 ou 5 horas. A forma inicial de tal comportamento específico é determinada, por exemplo, por um relógio que teve sua função discriminativa estabelecida (alterada) pela regra. Neste caso não se pode dizer que a resposta: "São 3 horas" esteja sob controle exclusivo do estímulo antecedente verbal: "Que horas são?", porque a sua forma não foi determinada exclusivamente pelo estímulo antecedente verbal.

No presente estudo não há um exemplo claro como esse, visto que os estímulos ou especificavam o comportamento a ser estabelecido (caso da instrução e da pergunta correspondente), ou especificavam o comportamento geral de apontar (caso da instrução e da pergunta mínima). Desse modo, pode-se dizer, por exemplo, que a instrução mínima funcionou como regra para o comportamento geral de apontar com o dedo para os estímulos de comparação, uma vez que ela estabeleceu que o participante deveria apontar, e não emitir outro comportamento qualquer, e estabeleceu este comportamento antes de ele ser afetado por suas consequências.

A instrução mínima também poderia ser classificada como regra porque ela estabeleceu a função discriminativa dos estímulos de comparação para o comportamento geral de apontar. Pode-se dizer que a instrução mínima exerceu essa função por três razões: 1) ela especificava que os participantes deveriam apontar para cada um dos três objetos de comparação e não para outro objeto qualquer presente na sala experimental; 2) antes de sua introdução, os participantes não estavam apontando para os três objetos de comparação; e, 3) depois de sua introdução, os participantes passaram a apontar para cada um dos três objetos de comparação.

Mas, como já dito, o comportamento específico que se seguiu à apresentação da instrução mínima não estava sob controle dessa instrução, uma vez que ela não estabeleceu a forma de tal comportamento. A instrução mínima também não estabeleceu as funções específicas das dimensões dos estímulos modelo e de comparação.

Diferentemente, a instrução correspondente estabeleceu tanto a forma do comportamento que se seguiu a sua apresentação, quanto as funções dos estímulos na presença dos quais esse comportamento ocorria. Por exemplo, na primeira sessão da Fase 2 do Participante P11, a instrução correspondente estabeleceu a sequência CEF e essa sequência foi mantida na segunda sessão dessa fase, apesar de não mais produzir o reforço programado. A instrução correspondente também estabeleceu que as dimensões do estímulo modelo e dos estímulos de comparação deveriam funcionar como estímulos discriminativos para as respostas de escolha na ordem: primeiro a dimensão cor, depois a dimensão espessura e, em seguida, a dimensão forma. Quando a pergunta correspondente foi apresentada na Fase 4, esta pergunta também estabeleceu tanto a forma inicial do comportamento (a sequência EFC), quanto a ordem em que as dimensões do estímulo modelo e dos estímulos de comparação deveriam funcionar como estímulos discriminativos para as respostas de escolha.

Isto pode ser afirmado porque, no caso de P11, por exemplo, tanto as sequências emitidas quanto as funções das dimensões dos estímulos foram alteradas de acordo com as mudanças das regras nas transições da Fase 1 para a Fase 2 e da Fase 3 para a Fase 4. Os arranjos de estímulos (cada um constituído de um estímulo modelo e três estímulos de comparação) não poderiam explicar as diferenças encontradas nos resultados, porque os arranjos usados não foram alterados entre as fases, visto que os arranjos usados na primeira fase foram os mesmos usados nas demais fases. As consequências programadas também não poderiam explicar as diferenças encontradas nos resultados, porque no início de cada fase as sequências foram emitidas, de acordo com as regras, antes de as consequências programadas poderem exercer algum efeito.

Esta análise sugere que a instrução correspondente exerceu um número maior de efeitos que a instrução mínima. De um modo mais geral, sugere que a abrangência dos efeitos exercidos por uma regra depende, em parte, do número de efeitos por ela descritos. Por essa visão, uma regra que especifica que os comportamentos A, B e C devem ser emitidos, por exemplo, teria maior chance de estabelecer tais comportamentos do que uma regra que não especifica comportamento.

Em síntese, os resultados do presente estudo sugerem que os efeitos de regras sobre o comportamento dependem, em parte, das propriedades formais das próprias regras e, portanto, tais propriedades deveriam ser consideradas como um fator na explicação do comportamento de seguir regras. Sugerem ainda que o termo regra poderia continuar sendo definido como estímulos antecedentes verbais (Hayes, 1986; Zettle \& Hayes, 1982), desde que isso não implique que os efeitos de regras ocorrem independentemente de suas propriedades formais, visto que apenas os estímulos que especificam 
o comportamento a ser estabelecido podem exercer a função de estabelecer comportamentos novos. Similarmente, o termo regra também poderia continuar sendo definido como estímulos antecedentes verbais especificadores de contingências (Skinner, 1966, 1969), mas, neste caso, deveria ficar claro que, além de estabelecer comportamentos, regras também podem alterar as funções de estímulos e para exercer essa função não é necessário que elas especifiquem claramente contingências.

Deste modo, seja qual for a definição usada, deveriam ser considerados os seguintes critérios para a definição de regras e do comportamento controlado por regras: a) para um estímulo antecedente ser classificado como regra ele deve poder alterar as funções dos estímulos e/ou estabelecer um comportamento; b) para estabelecer um comportamento é necessário que o estímulo antecedente verbal especifique o comportamento a ser estabelecido. Neste caso, o comportamento que se segue à apresentação da regra poderia ser controlado por regra, porque uma das exigências para um comportamento ser chamado de controlado por regras é que sua forma seja determinada pela regra; e, c) para alterar a função de estímulos não é necessário que o estímulo antecedente verbal especifique o comportamento a ser estabelecido e nem que especifique os estímulos a terem suas funções alteradas. Contudo, neste caso o comportamento específico que se segue à apresentação da regra não poderia ser chamado de puramente controlado por regras, uma vez que sua forma seria determinada por outra variável que não a regra que alterou a função do estímulo.

Pesquisas futuras deveriam tentar esclarecer como regras alteram que funções de estímulos e tentar identificar as condições sob as quais regras podem exercer essa função. Tais pesquisas seriam importantes, não apenas porque poucos estudos têm procurado investigá-las, mas também porque podem contribuir para esclarecer o controle por regras e a própria distinção entre o comportamento controlado por regras e o controlado por contingências.

Pesquisas futuras também deveriam continuar a investigação dos efeitos das propriedades formais dos estímulos verbais sobre o comportamento. Por exemplo, pesquisas futuras poderiam comparar os efeitos de um estímulo verbal apresentado na forma de uma ordem (de uma ameaça, de um aviso, etc) com os efeitos de um estímulo verbal apresentado na forma de uma sugestão (de uma súplica, de uma promessa, etc). Tais pesquisas poderiam contribuir para esclarecer que, além das variáveis históricas que antecedem o seguimento de regras e das consequências programadas para a ocorrência desse comportamento, os estímulos que constituem a regra, também podem interferir no seguimento de regras. Além disso, poderiam contribuir para identificar as propriedades definidoras de exemplos particulares de regras e os efeitos de tais estímulos sobre o seguimento de regras.

\section{Referências}

Albuquerque, L. C. (2001). Definições de regras. Em H. J. Guilhardi, M. B. B. P. Madi, P. P. Queiroz \& M. C. Scoz (Orgs.), Sobre comportamento e cognição: Expondo a variabilidade (pp.132140). Santo André: ARBytes.
Albuquerque, L. C. (2005). Regras como instrumento de análise do comportamento. Em L. C. Albuquerque (Org.), Estudos do comportamento (pp.143-176). Belém: Edufpa.

Albuquerque, L. C., \& Ferreira, K. V. D. (2001). Efeitos de regras com diferentes extensões sobre o comportamento humano. Psicologia: Reflexão e Crítica, 14, 143-155.

Albuquerque, L. C., Matos, M. A., de Souza, D. G., \& Paracampo, C. C. P. (2004). Investigação do controle por regras e do controle por histórias de reforço sobre o comportamento humano. Psicologia: Reflexão e Crítica, 17, 395-412.

Albuquerque, L. C., de Souza, D. G., Matos, M. A., \& Paracampo, C. C. P. (2003). Análise dos efeitos de histórias experimentais sobre o seguimento subseqüente de regras. Acta Comportamentalia, 11, 87-126.

Albuquerque, L. C., Reis, A. A., \& Paracampo, C. C. P. (2008). Efeitos de histórias de reforço, curtas e prolongadas, sobre o seguimento de regras. Acta Comportamentalia, 16, 305-332.

Barret, D. H., Deitz S. M., Gaydos G. R., \& Quinn P. C. (1987). The effects of programmed contingencies and social conditions on stereotype responses with human subjects. The Psychological Record, 34, 489-505.

Baron, A., \& Galizio, M. (1983). Instructional control of human operant behavior. The Psychological Record, 33, 495-520.

Braga, M. V. N., Albuquerque, L. C., \& Paracampo, C. C. P. (2005). Análise dos efeitos de perguntas e de instruções sobre o comportamento não-verbal. Interação em Psicologia, 9, 77-89.

Cerutti, D. T. (1989). Discrimination theory of rule-governed behavior. Journal of the Experimental Analysis of Behavior, 51, 259-276.

Cerutti, D. T. (1991). Discriminative versus reinforcing properties of schedules as determinants of schedule insensitivity in humans. The Psychological Record, 41, 51-67.

Cerutti, D. T. (1994). Compliance with instructions: Effects of randomness in scheduling and monitoring. The Psychological Record, 44, 259-269.

Galizio, M. (1979). Contingency-shaped and rule-governed behavior: Instructional control of human loss avoidance. Journal of the Experimental Analysis of Behavior, 31, 53-70.

DeGrandpre, R. J., \& Buskist, W. F. (1991). Effects of accuracy of instructions on human behavior: Correspondence with reinforcement contingencies matters. The Psychological Record, 41, 371-384.

Degrandpre, R., Buskist, W., \& Cush, D. (1990). Effects of orienting instructions on sensitivity to scheduled contingencies. Bulletin of Psychonomic Society, 28, 3311-334.

England, D. E., \& Buskist, W. (1995). The effects of instructions on subject's disclosure of information about operant tasks. The Psychological Record, 45, 451-461.

Hayes, S. C. (1986). The case of the silent dog-verbal reports and the analysis of rules: A review of Ericsson and Simon's "Protocol Analysis: Verbal reports as data." Journal of Experimental Analysis of Behavior, 45, 351-363.

Hayes, S. C., Brownstein, A. J., Zettle, R. D., Rosenfarb, I., \& Korn, Z. (1986). Rule governed behavior and sensitivity to changing consequences of responding. Journal of the Experimental Analysis of Behavior, 45, 237-256.

Joyce, J. H., \& Chase, P. N. (1990). Effects of response variability on the sensitivity of rule-governed behavior. Journal of the Experimental Analysis of Behavior, 54, 251-262. 
LeFrancois, J. R., Chase, P. N., \& Joyce, J. (1988). The effects of variety of instructions on human fixed-interval performance. Journal of the Experimental Analysis of Behavior, 49, 383-393.

Malott, R. W. (1989). The achievement of evasive goals: Control by rules describing contingencies that are not direct acting. Em S. C. Hayes (Eds.), Rule governed behavior: Cognition, contingencies, and instructional control (pp. 269-322). New York: Plenum.

Martinez, H., \& Tomayo, R. (2005). Interactions of contingencies, instructional accuracy, and instructional history in conditional discrimination. The Psychological Record, 55, 633-646.

Navarick, D. J. (2004). Analysis of impulsive choice: Assessing effects of implicit instructions. The Psychological Record, 49, 663-684.

Newman, B., Buffington, D. M., \& Hemmes, N. S. (1995). The effects of schedules of reinforcement on instruction following. The Psychological Record, 45, 463-476.

Otto, T. L., Torgrud, L. J., \& Holborn, S. W. (1999). An operant blocking interpretation of instructed insensitivity to schedule contingencies. The Psychological Record, 49, 663-684.

Paracampo, C. C. P., Albuquerque, L. C., Farias, A. F., Carvalló, B. N., \& Pinto, A. R. (2007). Efeitos de conseqüências programadas sobre o comportamento de seguir regras. Interação em Psicologia, 11, 161-173.

Pinto, A. R., Paracampo, C. C. P., \& Albuquerque, L. C. (2006). Análise do controle por regras em participantes classificados de flexíveis e de inflexíveis. Acta Comportamentalia, 14, 171-194.

Schlinger, H., \& Blakely, E. (1987). Function-altering effects of contingency-specifying stimuli. The Behavior Analyst, 10, 41-45.
Shimoff, E., Catania, A. C., \& Matthews, B. A. (1981). Uninstructed human responding: Sensitivity of low-rate performance to schedule contingencies. Journal of the Experimental Analysis of Behavior, 36, 207-220.

Skinner, B. F. (1963). Operant behavior. American Psychologist, 18, 503-515.

Skinner, B. F. (1969). Contingencies of reinforcement: A theoretical analysis. New York: Appleton-Century-Crofts.

Skinner, B. F. (1974). About behaviorism. New York: Alfred A. Knopf.

Torgrud, L. J., \& Holborn, S. W. (1990). The effects of verbal performance descriptions on nonverbal operant responding. Journal of the Experimental Analysis of Behavior, 54, 273-291.

Weiner, H. (1970). Instructional control of human operant responding during extinction following fixed-ratio conditioning. Journal of the Analysis Experimental of Behavior, 13, 391-394.

Zettle, R. D., \& Hayes, S. C. (1982). Rule-governed behavior: A potential theoretical framework for cognitive-behavior therapy. Em P. C. Kendall (Ed.), Advances in cognitive-behavioral research and therapy (pp. 73-118). New York: Academic Press.

Recebido em 22.06.09

Primeira decisão editorial em 20.10.09

Versão final em 13.11.09

Aceito em 13.04.10 